\title{
Meibomian Gland Dysfunction: A Dermatological Perspective on Pathogenesis and Treatment Outlook
}

\author{
Preeya K Gupta' \\ Laura M Periman ${ }^{2}$ \\ Edward Lain ${ }^{3}$ \\ Eric Donnenfeld (iD) ${ }^{4}$ \\ John Hovanesian (iD ${ }^{5}$ \\ Terry Kim ${ }^{6}$ \\ William Trattler ${ }^{7}$ \\ Elizabeth Yeu (1D ${ }^{8}$ \\ Edward Holland ${ }^{9}$
}

'Triangle Eye Consultants, Durham, NC, USA; ${ }^{2}$ Periman Eye Institute, Seattle, WA, USA; ${ }^{3}$ Sanova Dermatology, Pflugerville, TX, USA; ${ }^{4}$ Ophthalmic Consultants of Long Island, Garden City, NJ, USA; ${ }^{5}$ Harvard Eye Associates, Laguna Hills, CA, USA; ${ }^{6}$ Department of Ophthalmology, Duke University Eye Center, Durham, NC, USA; ${ }^{7}$ Center for Excellence in Eye Care, Miami, FL, USA; ${ }^{8}$ Virginia Eye Consultants, Norfolk, VA, USA; ' Cincinnati Eye Institute,

Edgewood, KY, USA
Correspondence: Preeya K Gupta Email preeyakgupta@gmail.com

\begin{abstract}
Meibomian glands are modified oil-producing glands that produce meibum and can become dysfunctional and negatively affect the lipid layer in the tear film, resulting in ocular surface diseases such as evaporative dry eye. Abnormal keratin production and aggregation at the meibomian gland orifice has been implicated in the pathogenesis of meibomian gland dysfunction (MGD). Current treatments largely ignore the role of keratin proteins. This review paper synthesizes various publications on hyperkeratinization and its role in MGD pathogenesis and proposes a novel treatment strategy for MGD that involves the use of keratolytic agents commonly used in dermatological treatments.
\end{abstract}

Keywords: meibomian gland disease, MGD, hyperkeratinization

\section{Introduction}

The first formal attempt to define and classify dry eye disease (DED) was the publication of a report by the National Eye Institute/Industry Workshop on Clinical Trials in Dry Eye in 1995, which named Meibomian gland dysfunction (MGD) as a key contributor to evaporative dry eye. ${ }^{1}$ Despite the role of MGD as a leading cause of evaporative DED throughout the world, there was no consensus on the definition, classification, diagnosis, or therapy of MGD until the International Workshop on Meibomian Gland Dysfunction published its initial report in 2011. ${ }^{2}$ The authors proposed the following formal definition of MGD:

MGD is a chronic, diffuse abnormality of the meibomian glands, commonly characterized by terminal duct obstruction and/or qualitative/quantitative changes in the glandular secretion. This may result in alteration of the tear film, symptoms of eye irritation, clinically apparent inflammation, and ocular surface disease. ${ }^{3}$

Much is still to be learned about MGD, including the influence of keratin in meibum and the gland structure, as well as the causes of hyperkeratinization. Despite the evidence of the role of keratinization in the obstruction of the glands, traditional MGD therapies have focused on removing the obstruction rather than addressing any underlying cause or pathology. This paper reviews the underlying pathophysiology of MGD and, given the vast similarities between Meibomian glands and other sebaceous glands, proposes adoption of dermatologic strategies to address a potential root cause of the obstructions. 


\section{Meibomian Gland Dysfunction Pathogenesis}

The central duct of the meibomian gland has been described as a "hair follicle without a hair shaft", ${ }^{4}$ as meibomian glands share many similarities with sebaceous glands with respect to embryologic development, structure, and the ability to undergo keratinization. ${ }^{4}$ An individual meibomian gland is made up of multiple acini that are arranged around a long central duct. ${ }^{5}$ Just as lipids are produced in sebaceous glands, meibomian glands produce meibum from whole glandular cells, requiring continuous cell turnover, production of new cells, and differentiation between cells within the acinus. ${ }^{4}$ Meibum is a complex mixture consisting of hundreds of different lipids, most of which are wax and cholesteryl esters, as well as 90 different proteins including various forms of keratin. ${ }^{6-8}$ Meibum is synthesized continuously inside the acini, and mechanical pressure created by blinking drives the meibum towards the terminal part of the ductal system and delivers the meibum onto the surface of the lid margin. The central duct through which the meibum passes is lined with modified keratinized epithelial cells while the gland orifice is located on the lid margin and is covered by epidermis. $^{9}$

Hyperkeratinization of the meibomian structure, resulting in obstructions and thickened meibum containing keratinized cell material, is considered to be a primary cause of meibomian gland dysfunction. ${ }^{3}$ Keratin is the end result of the transformation of epithelial cells, referred to as keratinocytes, once they have differentiated to form a horny cell layer such as that which occurs in skin, horns, nails, and hair tissue. However, in hyperkeratinization, this process is disturbed because of an excess of keratin formation and accumulation due to lack of adequate desquamation. ${ }^{10}$ This process is extremely wellstudied in skin and has been found to be a common mechanism in skin disorders.

Incorporation of as little as $10 \%$ additional keratin protein into meibum can increase the surface pressure of the meibum, making it more rigid and subject to fracture if incorporated into the lipid layer of the tear film. ${ }^{11,12}$ In addition, this raises the melting point of meibum so that it solidifies at body temperature and contributes to increased meibum viscosity and stasis. ${ }^{3,13,14}$ An increase and/or alteration in the meibum is cited as a cause for the degradation of the tear film and positively associated with severity of MGD., 3,14
Korb and Henriquez demonstrated hyperkeratinization at the Meibomian gland orifice as a cause of obstructive MGD in a series of patients who manifested with contact lens intolerance. ${ }^{15}$ Jester and colleagues validated this claim by identifying keratin proteins in the human Meibomian gland duct epithelial cells and determining that these cells were committed to the process of keratinization. ${ }^{16}$ Korb and Henriquez further elucidated the pathogenesis by reporting that the presence and characteristics of MGD were possible in symptomatic and nonsymptomatic patients whose eyelid margins do not have significant inflammation. ${ }^{15}$ Manual expression of the meibomian glands of these patients verified a non-obvious obstruction of the orifices and revealed clusters consisting of desquamated epithelial cells and thickened meibum. Histology of the obstructed glands showed dilation of the central duct by cell debris and sebaceous material.

\section{The Hyperkeratinization Process}

In healthy tissue, keratin crosslinking via disulfide bond formation is more likely to occur when a single protein has reached its intended final location. However, under oxidizing conditions, disulfide bond formation will occur in a less controlled manner and lead to the formation of aggregates in physiologically uncontrolled locations. Given the presence of reactive oxygen species in MGD, it may be that other oxidative processes, such as the formation of disulfide bonds, will be promoted in the disease state. Studies have demonstrated the contribution of oxidative stress to the pathophysiology of MGD, giving creed to this conclusion. In particular, proteome analysis of conjunctival epithelium tissue taken from patients suffering from MGD shows upregulation of proteins including peroxiredoxin 2 and 6 and heat shock protein 90 alpha (HSP90 $\alpha$ ). Peroxiredoxins are produced to control oxidation stemming from hydrogen peroxide and lipid peroxides, indicating an oxidative environment. Furthermore, HSP90 $\alpha$ is a molecular chaperone that has been shown to bind and protect the proteasome from oxidative inactivation amongst other functions. ${ }^{17}$ Preclinical studies also support the role of reactive oxygen species in MGD. For example, superoxide dismutase 1 (SOD1) knockout mice are observed to experience many of the biochemical and structural changes found in MGD, suggesting a role for oxidative stress in the disease pathology. ${ }^{18}$

Incorporation of keratin into meibum can increase the surface pressure of meibum above that of normal meibum with as little as a $10 \%$ increase in keratin protein making 
lipids more rigid and subject to fracture if incorporated into the lipid layer of the tear film. ${ }^{3,12}$ Together, these effects raise the melting point of meibum so that it solidifies at body temperature and contributes to increased meibum viscosity and stasis. $3,13,19$

\section{Limitations of Current Approaches to MGD Treatment}

Blackie and colleagues ${ }^{14}$ published a review of the known data in 2010, including Korb and Henriquez's study. The authors sought a paradigm shift in the understanding of MGD stating,

\begin{abstract}
Despite the potentially coexisting roles of bacteria, tear film osmolarity, and inflammation, it is critical to recognize that the primary mechanism for obstructive MGD is keratinization of the meibomian gland ducts. Thus, the success of any therapy for all forms of obstructive MGD is primarily dependent upon relief of the obstruction and secondarily upon the management of other factors such as inflammation and/or infection.
\end{abstract}

The primary aims of MGD treatment are to restore the normal flow and quality of meibomian gland secretions, thereby increasing the likelihood of a healthy lipid layer and stable tear film, which can be achieved through removal of obstructing material and the restoration of meibum secretion. ${ }^{19}$ Conventional methods for physical removal of material from the glands include the application of warm compresses and gentle lid massages to the eyelids, physical debridement of material along the lid margin, meibomian gland probing, as well as the use of thermal pulsation devices and microblepharoexfoliation. The effectiveness of thermal-based approaches for MGD treatment has been shown in the literature with respect to improving patient symptoms and relieving obstruction. ${ }^{20}$ With respect to modification of keratin, these approaches are less well studied and unlikely to impact keratin, as breaking the disulfide bridges that hold keratinized material together requires a temperature of approximately $145^{\circ} \mathrm{C}$, which would be unsafe for human application. While meibum release may be facilitated through the orifice distal to the obstruction in a gland, the use of heat and pressure to force meibum out from behind an unyielding fixed obstruction can increase intraductal pressure and rupture acini, forcing meibum into the lid tissue. This creates inflammation behind the obstruction, with the possibility of micro-injury to the delicate gland structure or ductal area leading to increased symptoms and secondary proximal atrophy, analogous to squeezing an unyielding acne blemish. $^{21}$

\section{Keratolytic Agents for Dermatologic Conditions}

To test the hypothesis that treatment of MGD requires addressing hyperkeratinization, it would be useful to consider keratolytic agents since they have proven to be effective for treating hyperkeratotic conditions of the skin. Keratolytic agents used in dermatology soften, separate, and cause desquamation of the cornified epithelium. Certain agents act on the corneocytes while others break down the lipid bilayer. Keratolytic agents include acids that hydrolyze keratins and disulfide bond disruptors that break apart crosslinked keratin.

Keratolytics commonly used in dermatology include alpha hydroxy acids (AHAs) and beta hydroxy acids (BHAs). AHAs are water-soluble, organic acids that reduce inter-corneocyte bonds by increasing the distance between corneocytes through the following mechanisms: increasing the stratum corneum water content, reducing the charges on the surface of the cells, inhibiting enzymes involved in the cohesion between corneocytes, and breaking desmosomes as they diminish the $\mathrm{pH}$ of the medium. Examples of AHAs include glycolic and lactic acids and they are applied topically to the skin in products like moisturizers, cleaners, masks, and other similar products. $^{22}$ Commonly used BHAs, such as Salicylic acid, are lipid-soluble, organic acids that reduce intracellular cohesion between corneocytes by dissolving intercellular cement material and reducing the $\mathrm{pH}$ of the stratum corneum, thereby increasing hydration and softening.

Selenium sulfide is a potent keratolytic agent that has a different mechanism of action than AHAs and BHAs. Selenium sulfide decreases the differentiation of epidermal cells and minimizes corneocyte production by slowing the production and development of keratinocytes. ${ }^{23}$

Selenium was first approved for medical use in the United States in $1951 .^{24}$ It is included on the World Health Organization's List of Essential Medicines, the most effective and safe medicines needed in a health system. Selenium sulfide is sold as an antifungal agent in shampoos for the treatment of dandruff and seborrheic dermatitis on the scalp with Malassezia genus fungi (ie, Selsun Blue, Head and Shoulders Clinical Strength, Exsel, Selsun, and Seleen). In the United States, a suspension with a detergent as a shampoo at $1 \%$ strength is available 
over the counter, and a $2.5 \%$ strength is also available with a prescription. At the $2.5 \%$ strength, selenium sulfide is also used on the body to treat tinea versicolor, a type of fungal skin infection caused by a different species of Malassezia.

It should be noted that off-label use of dermal keratolytics for ocular conditions is not recommended.

\section{Keratolytic Agents for Ocular Conditions}

Early studies demonstrated that selenium sulfide applied to the lid margin may be a successful treatment for seborrheic blepharitis. ${ }^{25-28}$ Recently, results of new studies of a novel drug candidate containing selenium sulfide have been presented. An interim analysis of a phase $2 \mathrm{a}$ multicenter, double-masked randomized controlled trial ${ }^{29}$ of AZRMD-001 (Azura Ophthalmics, Tel Aviv, Israel) looked at results of sequentially increasing concentrations $(0.1 \%$, $\mathrm{n}=9 ; 0.5 \%, \mathrm{n}=7 ; 1.0 \%, \mathrm{n}=7)$ or vehicle $(\mathrm{n}=9)$ of the ophthalmic ointment dosed twice weekly for up to 3 months in patients with mild to moderate MGD. Both the $0.5 \%$ and the $1.0 \%$ treatments showed significant improvement in the signs (MGYLS and MGS) and symptoms (Total ODSI score) of MGD compared to vehicle at the primary, Month 3 endpoint.

This dosing trial was followed by a study of $0.5 \%$ AZR-MD-001 ointment in patients with $\mathrm{MGD}^{30}{ }^{30}$ and a study of $1.0 \%$ AZR-MD-001 ointment in symptomatic contact lens wearers. ${ }^{31}$ In both studies, the selenium sulfide-containing ointment was found to restore gland function, improve eye dryness, and in the study of symptomatic contact lens wearers, improve symptoms such as fluctuating vision and increase comfortable wear time, without producing serious adverse events.

These positive findings with a selenium sulfidecontaining ointment are consistent with the role selenium sulfide plays in targeting aberrant keratins and disrupting disulfide bonds of the keratin plaques that can occur in both the meibum matrix and at the gland orifice, to unblock the glands. While many more studies are needed, this is an important step in demonstrating that addressing the root cause of MGD, hyperkeratinization, may bring relief.

\section{Conclusion}

MGD is a major cause of ocular surface diseases such as evaporative DED. Lipid composition and alteration in protein (eg keratin) production, deposition and aggregation have been implicated in the pathogenesis of MGD. Historically, there has been an emphasis on the increased melting point of meibum lipids. As such, therapeutic approaches have been targeted at lipid liquefaction along with expulsion of stagnated lipids in the gland's canal using therapies ranging from home lid warming and massage to more sophisticated and standardized approaches such as in-office warming and/or massage devices (eg, Lipiflow and iLux thermal pulsation).

While keratin proteins have been postulated to play a role in the pathophysiology of MGD, their exact contribution has largely been ignored and understudied. Abnormal keratinization of the gland orifice, central ducts and within the meibum of the meibomian glands may all be important drivers of MGD. Similar to dermatological disorders such as acne, MGD may start with retention hyperkeratosis, altered meibum flow, and then formation of a plug. Important new studies demonstrate that keratin modification via selenium sulfide may offer a novel pathway for therapeutic intervention in MGD. Greater understanding of the primary role of hyperkeratinization in MGD pathogenesis and the unique mechanism of action of selenium sulfide-containing therapeutics warrant further investigation.

\section{Ethics Approval/ Data}

As this is a prospective paper, there was no research conducted on humans or animals and no ethics approval was required, not is there any data associated with this paper.

\section{Acknowledgments}

The authors acknowledge writing and manuscript preparation assistance from Adrianne S. Resek, MA and Aron D. Ross, PhD, which assistance was funded by Azura Ophthalmics.

\section{Disclosure}

All authors of this paper meet the IMCJE authorship guidelines and take responsibility for the content of this paper. The following authors report they are paid consultants for Azura Ophthalmics: Preeya K Gupta, Laura M Periman, Edward Lain, Terry Kim, John Hovanesian, Edward Holland, William Trattler, Sheri Rowen, and Elizabeth Yeu. They were not paid for their contributions to this paper. Eric Donnenfeld reports personal fees from Tarsus, during the conduct of the study. John Hovanesian also reports equity interest from Tarsus, grants and/or personal fees from Novartis, Sun 
Pharma, and Allergan, during the conduct of the study. Terry Kim is also a consultant for Aerie Pharmaceuticals, Alcon, Allergan, Avedro, Avellino Labs, Bausch \& Lomb, CorneaGen (ownership as well), Dompe, Eyenovia (ownership as well), Johnson \& Johnson, other from 12. Kala Pharmaceuticals (ownership as well), Novartis, Ocular Therapeutix (ownership as well), Oculis, Omeros (ownership as well), Presbyopia Therapies (ownership as well), Sight Sciences, Simple Contacts (ownership as well), Surface, and Zeiss. The authors report no other conflicts of interest in this work.

\section{References}

1. Lemp MA. Report of the National Eye Institute/Industry workshop on clinical trials in dry eyes. CLAO J. 1995;21(4):221-232.

2. Nichols KK. The international workshop on Meibomian gland dysfunction: introduction. Invest Ophthalmol Vis Sci. 2011;52:1917-1921. doi:10.1167/iovs.10-6997

3. Nichols KK, Foulks GN, Bron AJ, et al. The international workshop on Meibomian gland dysfunction: executive summary. Invest Ophthalmol Vis Sci. 2011;52(4):1922-1929. doi:10.1167/iovs.106997a

4. Knop E, Ludescher M, Knop N. Keratinization status and cytokeratins of the human Meibomian gland epithelium. Acta Ophthalmol. 2009;87:2232. doi:10.1111/j.1755-3768.2009.2232.x

5. Knop E, Knop N, Millar T, et al. The international workshop on Meibomian gland dysfunction: report on the subcommittee on anatomy, physiology, and pathophysiology of the Meibomian gland. Invest Ophthalmol Vis Sci. 2011;52(4):1938-1978. doi:10.1167/ iovs. 10-6997c

6. Green-Church KB, Butovich I, Willcox M, et al. The international workshop on Meibomian gland dysfunction: report of the subcommittee on tear film lipids and lipid-protein interactions in health and disease. Invest Ophthalmol Vis Sci. 2011;52(4):1979-93. doi:10.1167/ iovs.10-6997d

7. Butovich IA. The Meibomian puzzle: combining pieces together. Prog Retin Eye Res. 2009;28(6):483-498. doi:10.1016/j. preteyeres.2009.07.002

8. Tsai PS, Evans JE, Green KM, et al. Proteomic analysis of human Meibomian gland secretions. Br J Ophthalmol. 2006;90(3):372-377. doi:10.1136/bjo.2005.080846

9. Foulks GN, Bron AJ. Meibomian gland dysfunction: a clinical scheme for description, diagnosis, classification, and grading. Ocul Surf. 2003;1(3):107-126. doi:10.1016/S1542-0124(12)70139-8

10. Deo PN, Deshmukh R. Pathophysiology of keratinization. J Oral Maxillofac Path. 2018;22(1):86-91.

11. Jester JV, Parfitt GJ, Brown DJ. Meibomian gland dysfunction: hyperkeratinization or atrophy? BMC Ophthalmol. 2015;15 (S1):3-11.

12. Ong BL, Hodson SA, Wigham T, et al. Evidence for keratin proteins in normal and abnormal human Meibomian fluids. Curr Eye Res. 1991;10(12):1113-1119. doi:10.3109/02713689109024128

13. Obata H. Anatomy and histopathology of human Meibomian gland. Cornea. 2002;21(7 Suppl):S70-S74.
14. Blackie CA, Korb DR, Knop E, et al. Nonobvious obstructive Meibomian gland dysfunction. Cornea. 2010;29(12):1333-1345. doi:10.1097/ICO.0b013e3181d4f366

15. Korb DR, Henriquez AS. Meibomian gland dysfunction and contact lens intolerance. J Am Optom Assoc. 1980;51:243-251.

16. Jester JV, Nicolaides N, Smith RE. Meibomian gland dysfunction. I. Keratin protein expression in normal human and rabbit meibomian glands. Invest Ophthalmol Vis Sci. 1989;30(5):927-935.

17. Soria J, Acera A, Durán JA, et al. The analysis of human conjunctival epithelium proteome in ocular surface diseases using impression cytology and 2D-DIGE. Exp Eye Res. 2018;167:31-43. doi:10.1016/ j.exer.2017.03.006

18. Ibrahim OM, Dogru M, Matsumoto Y, et al. Oxidative stress induced age dependent Meibomian gland dysfunction in $\mathrm{Cu}, \mathrm{Zn}$-superoxide dismutase-1 (Sod1) knockout mice. PLoS One. 2014;9(7):e99328. doi:10.1371/journal.pone.0099328

19. Shine WE, McCulley JP. Meibomianitis: polar lipid abnormalities. Cornea. 2004;23:781-783. doi:10.1097/01.ico.0000133995.99520.1f

20. Lane SS, DuBiner HB, Epstein RJ, et al. A new system, the LipiFlow, for the treatment of meibomian gland dysfunction (MGD). Cornea. 2012;31(4):396-404. doi:10.1097/ICO.0b013e318239aaea

21. Maskin SL, Testa WR. Growth of Meibomian gland tissue after intraductal Meibomian gland probing in patients with obstructive Meibomian gland dysfunction. $B r \quad J$ Ophthalmol. 2018;102 (1):59-68. doi:10.1136/bjophthalmol-2016-310097

22. Babilas P, Knie U, Abels C. Cosmetic and dermatologic use of alpha hydroxy acids. J German Soc Dermatol. 2012. doi:10.1111/j.16100387.2012.07939.x.

23. Gupta AK, Foley KA, et al. Topical antifungal agents. In: Comprehensive Dermatologic Drug Therapy. 4th ed. 2021.

24. AHFS Drug Information. Selenium Sulfide. McEvoy GK, editor. Bethesda, MD: American Society of Health-System Pharmacists; 2007:3521-3522.

25. Bahn GC. The treatment of seborrheic blepharitis. South Med J. 1954;47(8):749-753. doi:10.1097/00007611-195408000-00008

26. Thygeson P, Vaughan DG Jr. Seborrheic blepharitis. Trans Am Ophthalmol Soc. 1954-1955;52:173-188.

27. Cohen LB. Use of selsun in blepharitis marginalis. Am J Ophthalmol. 1954-1955;38(4):560-562. doi:10.1016/0002-9394(54)90708-4

28. Wong AS, Fasanaella RM, Haley LD, et al. Selenium (selsun) in the treatment of marginal blepharitis. AMA Arch Ophthalmol. 1956;55 (2):246-253. doi:10.1001/archopht.1956.00930030250011

29. Downie LE, Watson SL, Tan JL, Stapleton F, Bosworth C. A multicenter, double-masked, vehicle-controlled, randomized, parallel group clinical trial of AZR-MD-001 (Sterile Ophthalmic Ointment Selenium Sulfide $\mathrm{SeS}_{2}$ ) in individuals with Meibomian gland dysfunction. Presented at the Annual Meeting of the Association for Research in Vision and Ophthalmology; May 1 07; 2021. Virtual.

30. Craig J, Stapleton F, Tan J, et al. Randomized controlled trial evaluating novel keratolytic for MGD Treatment. Presented at the Annual Meeting of the Association for Research in Vision and Ophthalmology; May 1 07; 2021. Virtual.

31. Stapleton F, Tan J, Jia T, DePuy V, Gleeson M, Bosworth C The effect of a novel selenium sulfide-containing topical treatment on ocular signs and symptoms in symptomatic contact lens wearers: an exploratory study. Presented at the Annual Meeting of the Association for Research in Vision and Ophthalmology; May 107; 2021. Virtual. 


\section{Publish your work in this journal}

Clinical Ophthalmology is an international, peer-reviewed journal covering all subspecialties within ophthalmology. Key topics include: Optometry; Visual science; Pharmacology and drug therapy in eye diseases; Basic Sciences; Primary and Secondary eye care; Patient Safety and Quality of Care Improvements. This journal is indexed on PubMed

Submit your manuscript here: https://www.dovepress.com/clinical-ophthalmology-journal
Central and CAS, and is the official journal of The Society of Clinical Ophthalmology (SCO). The manuscript management system is completely online and includes a very quick and fair peer-review system, which is all easy to use. Visit http://www.dovepress.com/ testimonials.php to read real quotes from published authors. 